\title{
PW laser-driven bright $\gamma$-ray emission and dense positron production from diamondlike carbon foils
}

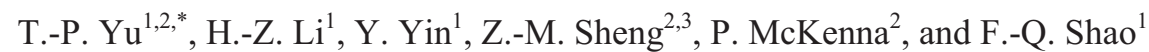 \\ ${ }^{1}$ Department of Physics, National University of Defense Technology, Changsha 410073, China \\ ${ }^{2}$ SUPA Department of Physics, University of Strathclyde, Glasgow G4 0NG, UK \\ ${ }^{3}$ College of Physics and Astronomy, Shanghai Jiao Tong University, Shanghai, 200240, China \\ *tongpu@nudt.edu.cn
}

\begin{abstract}
-we propose an all-optical scheme for ultra-bright $\mathrm{y}$ ray emission and dense positron production with lasers at intensity of $10^{22-23} \mathrm{Wcm}^{-2}$. By irradiating two colliding ellipticallypolarized lasers onto two diamondlike carbon foils, electrons in the focal region of one foil are rapidly accelerated by the laser radiation pressure and interact with the other intense laser pulse which penetrates through the second foil due to relativistically induced foil transparency. This symmetric configuration enables efficient y-photon emission with unprecedented brightness of $10^{25}$ photons $/ \mathrm{s} / \mathrm{mm}^{2} / \mathrm{mrad}^{2} / 0.1 \% \mathrm{BW}$ at $15 \mathrm{MeV}$ and intensity of $5 \times 10^{23} \mathrm{Wcm}^{-2}$. A GeV positron beam with density of $2.5 \times 10^{22} \mathrm{~cm}^{-3}$ and flux of $1.6 \times 10^{10} /$ shot is achieved.
\end{abstract}

Keywords- high power laser, Breit-Wheeler process, nonlinear Compton scattering, radiation pressure acceleration.

\section{INTRODUCTION}

Matter can be transferred into energy and the opposite transformation is also possible by use of high-power lasers. A laser pulse in plasma can convert its energy into $\mathrm{y}$-rays and then electron-positron pairs via the multi-photon Breit-Wheeler process. This is a totally unexplored research area, which opens up new avenues in high energy density physics, particle and nuclear physics, and high energy astrophysics.

\section{SCHMME AND SimULATIONS}

The open source QED-PIC code EPOCH is employed, which has incorporated the binary collision, $\gamma$-ray emission, radiation reaction, and pair production by both the trident process and multi-photon BW process. A Monte Carlo algorithm with quantum correction is implemented in the code for calculating the photon emission and pair production. Here, The trident process is switched off and the $\mathrm{BH}$ is also ignored because of the use of low- $\mathrm{Z}$ ultra-thin foils. For simplicity, the $\mathrm{e}^{-} \mathrm{e}^{+}$annihilation is ignored in the code.

The simulation box size is $\mathrm{X} \times \mathrm{Y} \times \mathrm{Z}=20 \lambda_{0} \times 14 \lambda_{0} \times 14 \lambda_{0}$. Here $\lambda_{0}=\mathrm{cT}_{0}=1 \mu \mathrm{m}$ is the laser wavelength and $\mathrm{T}_{0}=3.3 \mathrm{fs}$ is the laser cycle. The foil electron density is $n_{e}=200 n_{c}$, mixed with $20 \%$ protons in number density. Both foils have a thickness of $\mathrm{L}=0.32 \mu \mathrm{m}$ and radius of $\mathrm{R}=5 \mu \mathrm{m}$, as shown in Fig. 1. Two identical EP Gaussian laser pulses with intensity component of $\mathrm{a}_{\mathrm{y}}=237$ and $\mathrm{a}_{\mathrm{z}}=154$ and focal size of $\sigma_{0}=4 \mu \mathrm{m}$ irradiate the two foils simultaneously. Each pulse has a trapezoidal profile in time with a duration of $10 \mathrm{~T}_{0}$. Thus the total laser energy of each laser pulse is about $1600 \mathrm{~J}$.

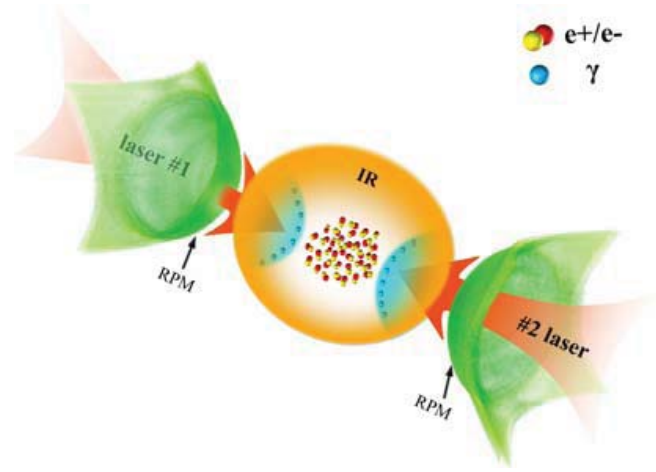

Fig. 1. Schematic diagram of bright $\gamma$-ray emission and dense pair production by counter-propagating lasers irradiating two diamondlike carbon (DLC) foils.

\section{RESULTS AND CONCLUSIONS}

It is shown that, by irradiating the two colliding ellipticallypolarized lasers onto two diamondlike carbon foils, electrons in the focal region of one foil are rapidly accelerated by the laser radiation pressure and interact with the other intense laser pulse which penetrates through the second foil due to relativistically induced foil transparency [1]. This symmetric configuration enables efficient Compton back-scattering and results in ultrabright $\gamma$-photon emission with brightness of $\sim 10^{25}$ photons/ $\mathrm{s} / \mathrm{mm}^{2} / \mathrm{mrad}^{2} / 0.1 \% \mathrm{BW}$ at $15 \mathrm{MeV}$ and intensity of $5 \times 10^{23}$ $\mathrm{Wcm}^{-2}$, and a $\mathrm{GeV}$ positron beam with density of $2.5 \times 10^{22}$ $\mathrm{cm}^{-3}$ and flux of $1.6 \times 10^{10} / \mathrm{shot}$. To the best of our knowledge, it the first time to report such high-brightness ultra-short $\mathrm{y}$-ray emission and dense positron production in full 3D configuration of laser-foil interaction [2]. Meanwhile, collective effects of the pair plasma may be also triggered. The laser intensity required is within the capabilities of future multi-PW laser facilities, paving the way to potential applications in nuclear and particle physics for fundamental research, laboratory study of astrophysics, medical imaging, and material science.

\section{REFERENCES}

[1] H.Z. Li, et al. "Ultra-bright $\gamma$-ray emission and dense positron production from two laser-driven colliding foils," Sci. Rep. vol. 7, pp 17132, December 2017.

[2] X.L. Zhu, et al. "Dense GeV electron-positron pairs generated by lasers in near-critical-density plasmas," Nat. Commun. vol. 7, pp. 13686, December 2016. 